\title{
¿Es correcto que en un número significativo de hospitales latinoamericanos, todavía se siga empleando comida licuada para nutrir a pacientes complicados?
}

Is it right that in a significant number Latin American hospitals still continue using liquified food to nourish a complicated patients?

Es incorrecto desde cualquier punto de vista, sin embargo, se hace. A pesar de que la pregunta puede parecer tonta, irrelevante y hasta capciosa, insuficiente desde cualquier ángulo como para motivar la construcción de una nota al respecto, en la actualidad se hace. A pesar de que hoy en día existe disponible a nivel comercial una variedad abrumadoramente grande de fórmulas para uso enteral, la comida licuada se sigue empleando para nutrir, vía sonda, a pacientes con patologías de diferentes grados de complejidad; en el colmo de los casos, se espera que el paciente se complique para, recién en ese momento, iniciar el uso de fórmulas especializadas.

La comida licuada es llamada de mil maneras: fórmula artesanal, fórmula institucional, fórmula alimentaria modificada, alimentación modificada nutricional y así sucesivamente, sin embargo todos estos términos hacen referencia a lo mismo, alimentos que han sido fraccionados y diluidos para pasar por una sonda, pero nada más. Con lo escrito, no queremos restarle importancia a la comida, lo que sucede es que el alimento posee características bioquímicas y nutricionales que no siempre son útiles para fortalecer con rapidez el Estado Nutricional de un paciente complicado.

La comida licuada no nos permite hacer modificaciones en la estructura bioquímica de los macronutrientes, sobre todo en el caso de la proteína, esta siempre será polimérica; en el mejor de los casos, con el licuado se consigue fraccionarla pero no digerirla. La fórmulas especializadas ponen a nuestra disposición, además de la proteína, lípidos y carbohidratos con diferente estructura bioquímica (polimérica, semi-elemental o elemental) (tabla 1). Esto permite que nuestra Nutrición se ajuste mejor a las características funcionales del Aparato digestivo del paciente. Por ejemplo, si tenemos pacientes con fallas en la liberación de bilis, debemos emplear Triglicéridos de cadena media (TCM) que no requieren bilis para ser emulsionados; no hay forma de encontrarlos en los alimentos porque son moléculas sintéticas (elaboradas en un laboratorio)(1).

Tabla 1. Características de los nutrientes según tipo de fórmula

\begin{tabular}{|c|c|c|c|}
\hline & Poliméricas & Oligomonoméricas & \\
\hline $\begin{array}{l}\text { Macronutrientes } \\
\text { Proteínas }\end{array}$ & $\begin{array}{l}\text { Caseinato sódico } \\
\text { Caseinato cálcico } \\
\text { Lactoalbúmina } \\
\text { Proteína de soja }\end{array}$ & $\begin{array}{l}\text { Peptídicas } \\
\text { Hidrolizado de caseína } \\
\text { Hidrolizado de soja } \\
\text { Hidrolizado de lactoalbúmina }\end{array}$ & $\begin{array}{l}\text { Elementales } \\
\text { Aminoácidos libres }\end{array}$ \\
\hline Lípidos & $\begin{array}{l}\text { Grasa animal } \\
\text { Aceite de canola } \\
\text { Aceite de maíz } \\
\text { Aceite de semilla de soja }\end{array}$ & $\begin{array}{l}\text { Aceite de girasol } \\
\text { Aceite de Coco } \\
\text { Aceite de maíz } \\
\text { Aceite de semilla de soja }\end{array}$ & TCM \\
\hline Carbohidratos & $\begin{array}{l}\text { Dextrinomaltosa } \\
\text { Polimeros de glucosa } \\
\text { Almidón de maíz } \\
\text { Sacarosa }\end{array}$ & $\begin{array}{l}\text { Hidrolizado de almidón } \\
\text { Oligosacáridos de glucosa }\end{array}$ & $\begin{array}{l}\text { Fructuosa } \\
\text { Sacarosa }\end{array}$ \\
\hline
\end{tabular}


La comida licuada no nos permite manipular con precisión la cantidad de proteína que se le brindará a un paciente sobre todo cuando sus necesidades son altas y/o existe restricciones para el consumo de uno u otro aminoácido. En la cirrosis, por ejemplo, el requerimiento de proteína no solo puede ser alto, sino que además la proteína debe proporcionar una mayor cantidad de aminoácidos ramificados que aromáticos, es decir, se debería priorizar el uso de proteína vegetal. ¿Ha pensado cuánto alimento se necesitaría para cubrir $90 \mathrm{~g}$ de proteína vegetal y cuáles son las posibilidades que un paciente complicado pueda comer ese volumen de alimento? Con una fórmula especializada, este requerimiento se puede cubrir con suma facilidad.

La comida licuada contiene elementos que pueden incrementar la osmolaridad del preparado final de manera importante, sin embargo, es prácticamente imposible determinar con precisión la osmolaridad de un licuado. La Osmolaridad es un escollo difícil de sortear cuando hablamos de alimentación intraintestinal. El intestino no admite osmolaridades altas (máximo 300mOsm) y la comida licuada por sus problemas de sedimentación suele ser administrada en bolos lo que incrementa el riesgo de diarrea osmótica (es difícil determinar hasta cuánto podría subir la osmolaridad de un licuado). Una manera de reducir la osmolaridad de la comida licuada es diluirla o proporcionar menor cantidad de la misma, con lo cual, el paciente suele recibir menos volumen del que necesitaría y por tanto, menos energía y nutrientes. Las fórmulas especializadas poseen una osmolaridad conocida que permite administrarlas con seguridad garantizando un mayor aporte nutricional

La comida licuada, sobre todo la administrada por sonda, debe tener una densidad energética menor a 1, es decir debe proporcionar como máximo 1 caloría por cada centímetro cúbico porque de otra manera no podría fluir con facilidad a través de la sonda. La densidad es un parámetro, particularmente importante en pacientes con demandas altas de energía y/o restricciones importantes en el volumen permitido como es el caso de los pacientes renales, cardiópatas, con fístulas o desnutrición. ¿Cómo haríamos para proporcionarle, solo con comida, $2000 \mathrm{kcal}$ a un paciente que no puede recibir más de 1000 cc por día? Peor aún, ¿Cómo administramos 2000 kcal a este mismo paciente si, además, tiene una restricción importante de proteína? Con comida licuada es imposible, sin embargo, contamos con fórmulas de densidad 2.0 y con aporte reducido de proteinas.

No obstante lo citado, la comida licuada se emplea y se emplea mucho más de lo que debería. Alguien podría indicar que usar una fórmula especializada es una herramienta facilista, sin embargo, no es así. Seleccionar la fórmula más adecuada y administrarla de manera apropiada es un tema que demanda mucha preparación y estudio: cuando se cuenta con estos dos aspectos la selección se hace sencilla y la recuperación del paciente se logra con rapidez; si no se cuenta con estos pilares, el empleo de una fórmula podría, por el contrario, complicar la situación clínica del paciente (3).

Qué ha pasado para que en pleno siglo XXI se sigan empleando los mismos productos que se empleaban hace más de 50 años. ¿Por qué se sigue empleando comida licuada? Es una respuesta difícil de responder. ¿No se conoce, acaso, suficiente sobre ellas? ¿No se sabe emplearlas? ¿Existe alguna especie de prejuicio o temor en relación a su uso? ¿Los propios Nutricionistas/Nutriólogos no estamos lo suficientemente convencidos que un paciente podría vivir y recuperarse consumiendo solo fórmulas especializadas? La priorización del uso de comida cuando el tracto gastrointestinal está integro no siempre es una buena decisión porque como hemos descrito líneas arriba la comida no nos permite todas las manipulaciones en cuanto al aporte nutricional que las fórmulas especializadas, si permiten.

El único objetivo de esta nota es hacer un llamado a la conciencia colectiva de los Nutricionistas/Nutriólogos acerca de la importancia del conocimiento y el uso de fórmulas especializadas de Nutrición Enteral. En las farmacias hospitalarias existe cada vez menos variedad de estos productos debido al poco requerimiento que se hace de las mismas, algo que es sumamente irónico, porque se ha demostrado por todo el mundo que es más barato brindar nutrición enteral 
durante 5 días que mantener hospitalizado a un pacientes durante un mes; se ha demostrado que mientras mejor es el Estado de Nutrición más rápido se da el alta; se ha demostrado que mientras mejor es el Estado de Nutrición, menor será la cantidad de antibióticos empleado; se ha demostrado que mientras mejor es el Estado de Nutrición hay menor riesgo de recaidas.

Finalmente y desde el punto de vista Ético, la obligación del Nutricionista/Nutriólogo (dependiendo de la denominación del país) es agotar todos sus esfuerzos para conseguir la mejor herramienta disponible para la recuperación de su paciente. Los Nutricionistas/Nutriólogos son profesionales cuya mayor virtud es tener la capacidad de convertir un requerimiento nutricional (gramos de esto o aquello) en una cantidad determinada de fórmula, alimento o suplemento. Lamentablemente, si nuestro convencimiento no es lo suficientemente fuerte como para exigir la presencia de formulas especializadas en los hospitales, la poca demanda de estos productos hará que paulatinamente desaparezcan del mercado, con lo cual, la recuperación de los pacientes se verá afectada dramáticamente. Recuerde que una fórmula bien elegida y bien administrada puede hacer que un paciente se recupere en cuestión de días, una fórmula mal seleccionada o el uso de comida licuada puede alargar innecesariamente el tiempo que requiera la recuperación de un paciente.

Se ha preguntado ¿Cuántos pacientes podrían estar siendo "desahuciados" cuando en realidad lo único que necesitan es una adecuada repleción Nutricional? Se ha preguntado icuántos de sus pacientes con cáncer, podrían haber tenido desenlace final menos dramático si se hubiesen encontrado bien nutridos? Estas líneas se han escrito con único objetivo de levantar la voz sobre un hecho concreto: el uso de fórmulas especializadas; dejemos de vivir en el mundo al revés, teniendo cerca herramientas tan potentes para salvar vidas, empleémoslas más intensamente; el paciente se lo agradecerá, literalmente con su vida.

\section{Referencias Bibliográficas}

1. Valenzuela B Alfonso, Sanhueza C Julio, Nieto K Susana. EL USO DE LíPIDOS ESTRUCTURADOS EN LA NUTRICIÓN: UNA TECNOLOGÍA QUE ABRE NUEVAS PERSPECTIVAS EN EL DESARROLLO DE PRODUCTOS INNOVADORES. Rev. chil. nutr. [revista en la Internet]. 2002 Ago [citado 2013 Ago 19] ; 29(2): 106-115. Disponible en: http://www.scielo.cl/scielo.php?script=sci_arttext\&pid=S0717-75182002000200005\&lng =es. http://dx.doi.org/10.4067/S0717-75182002000200005

2. Modificado de Del Olmo D. Productos dietéticos para usos nutricionales específicos. En: Planas M, Alvarez J, Culebras J, García. León M. Maldonado J. Mesejo A. Montejo J (ed). Tratado de Nutrición Tomo IV Nutrición Clínica de Angel Gil. Madrid: Médica Panamericana. 2010.

3. Cruz R, Herrera T. Procedimientos Clínicos para la Atención Nutricional en Hospitalización y en Consulta. 1ª Edición. Lima: Fondo Editorial IIDENUT. 2014.

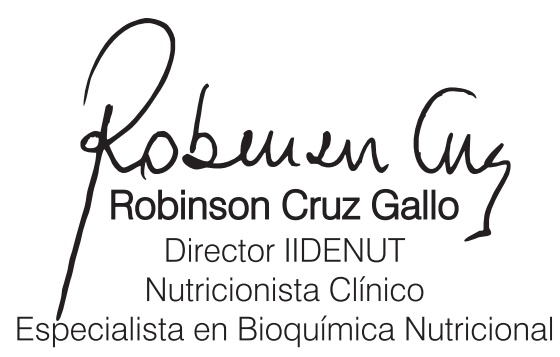

